\title{
Supplementary Information Unraveling the supramolecular organization mechanism of chiral star-shaped poly(3-alkylthiophene)
}

\author{
Michèle Moris $^{* 1}$, Marie-Paule Van Den Eede ${ }^{2}$, Guy Koeckelberghs², Olivier \\ Deschaume $^{3}$, Carmen Bartic ${ }^{3}$, Koen Clays ${ }^{1}$, Stijn Van Cleuvenbergen ${ }^{* 4}$ and Thierry \\ Verbiest $^{* 1}$ \\ ${ }^{1}$ Department of Chemistry, Molecular Imaging and Photonics, KU Leuven, Celestijnenlaan 200D, 3001 \\ Heverlee, Belgium. \\ 2 Department of Chemistry, Polymer Chemistry and Materials, KU Leuven, Celestijnenlaan 200F, 3001 \\ Heverlee, Belgium. \\ ${ }^{3}$ Department of Physics and Astronomy, Soft-Matter Physics and Biophysics Section, KU Leuven, \\ Celestijnenlaan 200D, 3001 Heverlee, Belgium. \\ ${ }^{4}$ Department of Chemistry, Physical Chemistry, KU Leuven-KULAK, Etienne Sabbelaan 53, 8500 \\ Kortrijk, Belgium.
}

\section{Supplementary Methods}

\section{SHS and THS setup}

A home-made harmonic scattering setup ${ }^{1}$ was used to measure THS, SHS and MPF. All signals could be measured simultaneously. Scattering was induced by a tunable Spectra-Physics Insight DeepSee femtosecond pulsed laser. The light was linearly polarized in the z-direction, perpendicular to the optical table and was focused in the polymer solution (in a $1 \mathrm{~cm}$ quartz cuvette). The beam waist was $\sim 8 \mu \mathrm{m}$ resulting in a Rayleigh length of $\sim 190 \mu \mathrm{m} .{ }^{1}$ The scattered light was collected at an angle of $90^{\circ}$ with an Andor iXon 897 EMCCD camera after passing through a Bruker SureSpectrum 500is spectrometer. A Wollaston prism split the scattered light in its vertically and horizontally polarized components allowing to obtain the depolarization ratio continuously. By using this spectral setup in combination with the CCD camera and curve fitting, contributions from MPF, SHS and THS to the whole spectrum could be resolved.

The absorbance and emission of the star-shaped P3AT is strong in the visible part of the spectrum. To avoid strong overlap of SHS and THS signals with multiphoton fluorescence, we opted for recording SHS and THS separately. For SHS an excitation wavelength of $860 \mathrm{~nm}$ was used. For THS we used an excitation wavelength of $1300 \mathrm{~nm}$. The data acquisition time was 2 seconds or was recalculated to match the time frame for correlation purposes.

The contribution of the (non-)solvents to the total scattering signal was always negligible compared to the contribution of the polymer. The SHS and THS intensities behave linearly with polymer concentration (Figure S1). 


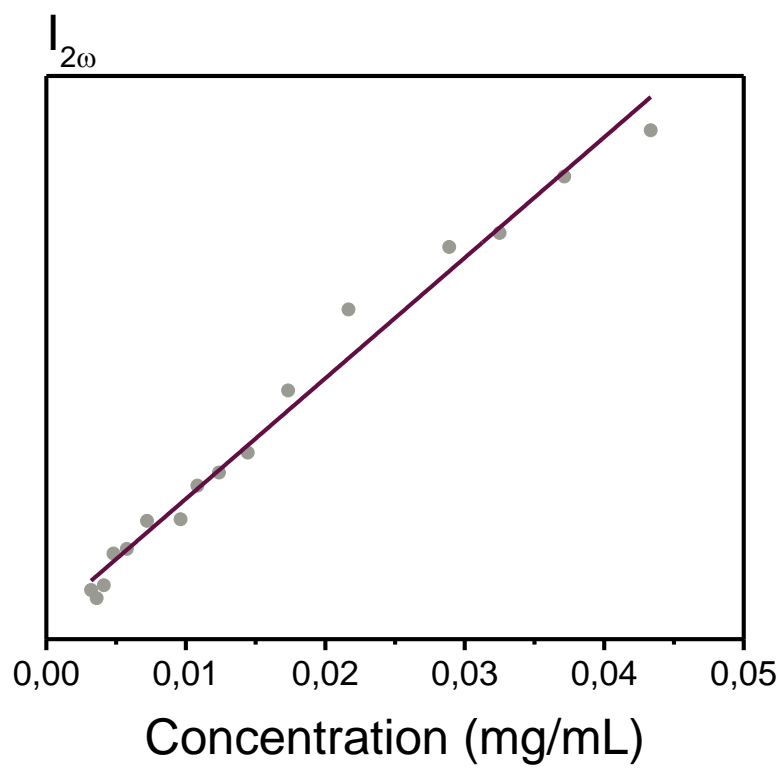

Figure S1: Concentration dependent SHS measurements of a $20 \%(\mathrm{v} / \mathrm{V})$ non-solvent/solvent solution of the starshaped P3AT. A linear behavior for the non-aggregated solute is observed.

\section{AFM}

For AFM analysis, a $5 \mu \mathrm{L}$ droplet of sample was deposited on a freshly cleaned silicon substrates freshly cleaned immediately followed by drying using compressed air to eliminate the influence of the solvent evaporation rate on the sample. Only fully grown assemblies were analyzed. A Bruker multimode 8 AFM system with MSNLF cantilevers $(f=110-120$ $\mathrm{kHz}, \mathrm{k}=0.6 \mathrm{~N} \mathrm{~m}^{-1}$, average tip radius of $2-12 \mathrm{~nm}$ ) was used for topographical imaging in PeakForce Tapping mode. The AFM topography images were leveled and line-corrected using Gwyddion. 


\section{Supplementary Results}

\section{Fluorescence spectra}

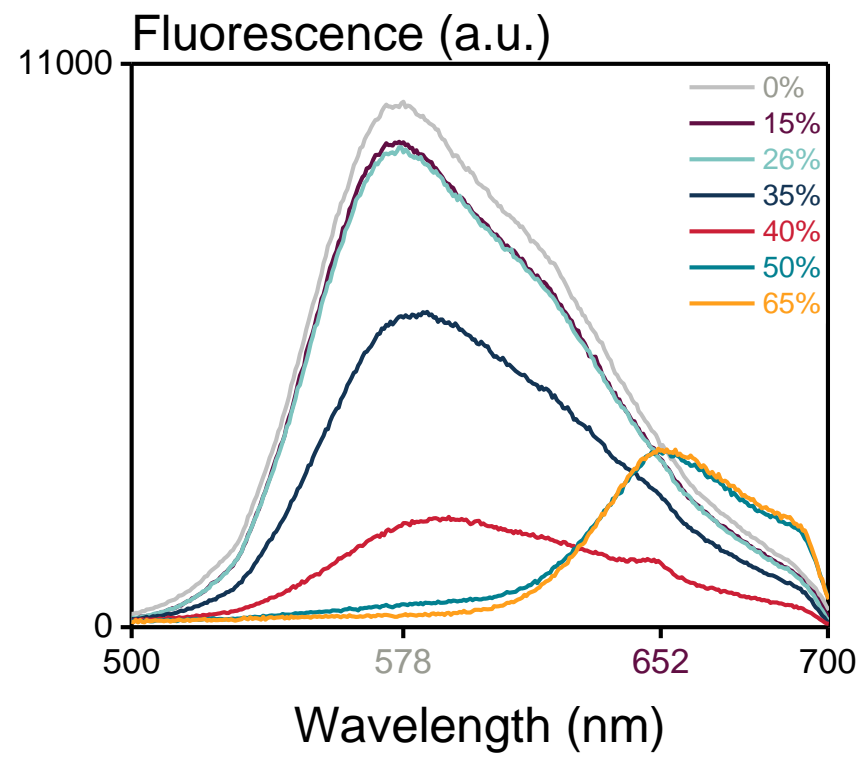

Figure S2: Discrete MPF spectra. The initial peak at $578 \mathrm{~nm}$ decreases upon addition of non-solvent, while a new peak at $652 \mathrm{~nm}$ appears once a supramolecular assembly has formed. The spectra are corrected for dilution.

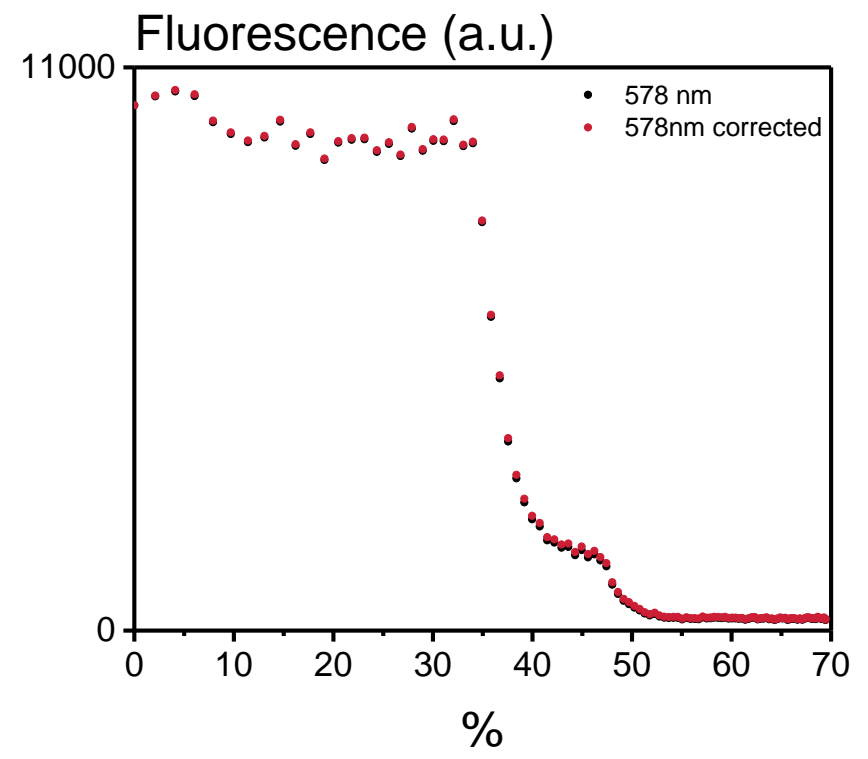

Figure S3: Dynamic fluorescence data at $578 \mathrm{~nm}$ corrected (red dots = corrected, black dots = not corrected) for the influence of absorption. ${ }^{2}$ There is no significant difference between both graphs.

\section{Absorption spectra}




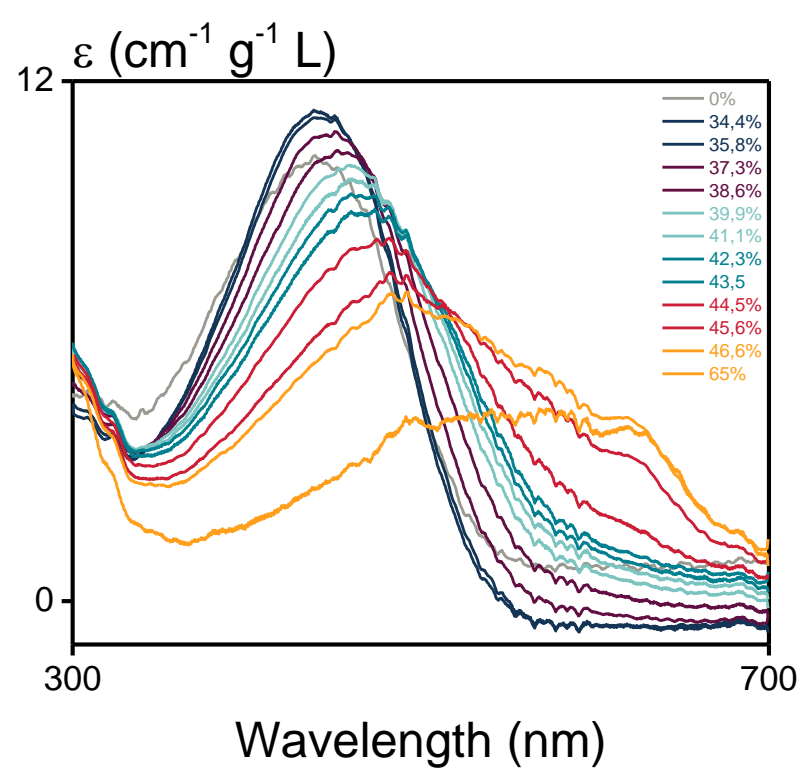

Figure S4: Absorption spectra for different amount of methanol added between 34 and 47\% (v/v). These spectra were recorded with a Jasco J-810 spectrometer simultaneously with CD measurements.

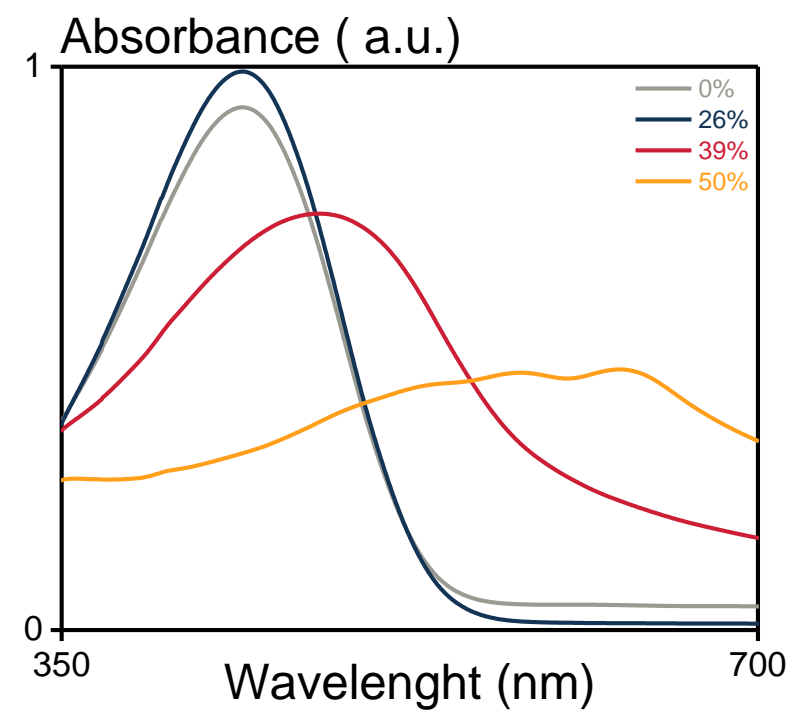

Figure S5: Discrete UV/Vis absorption spectra for a fixed polymer concentration $(0.0235 \mathrm{mg} / \mathrm{mL}$ at $0,26,39$ and $50 \%(\mathrm{~V} / \mathrm{V})$ of methanol). The absence of an isosbestic point is clearly observed. 


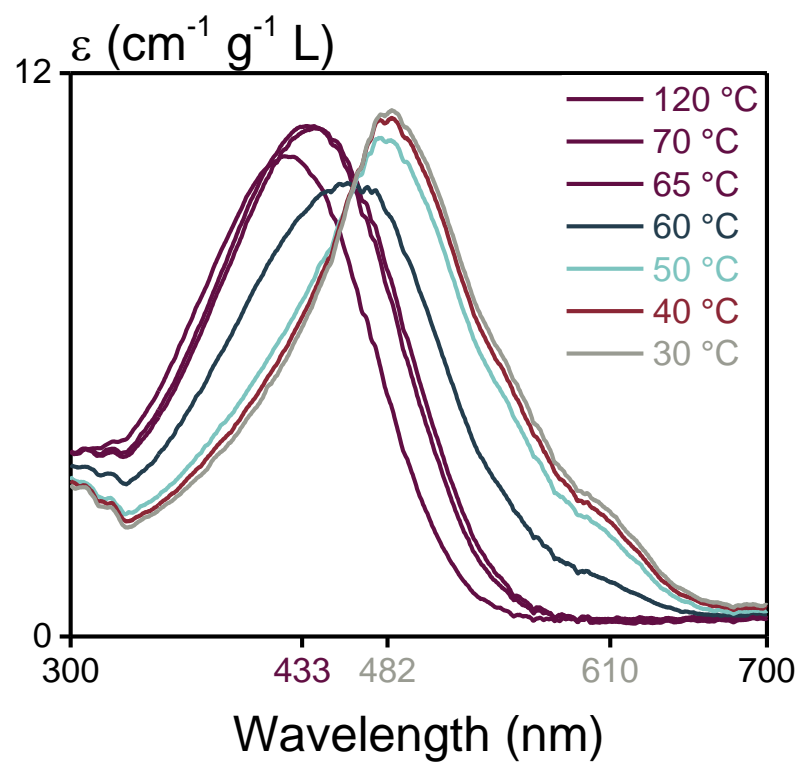

Figure S6: Discrete absorption spectra at several temperatures. These spectra were recorded with a Jasco J-810 spectrometer simultaneously with $C D$ measurements. For the three first spectra with similar color code (before self-assembly) the band with peak maximum at $433 \mathrm{~nm}$ is recorded at a solution temperature of $120^{\circ} \mathrm{C}$, while the other two that are slightly red-shifted are recorded at a solution temperature of $70{ }^{\circ} \mathrm{C}$ and $65^{\circ} \mathrm{C}$, right before selfassembly sets in. The small shift is probably due to the presence of a very small amount of already formed assemblies.

\section{DLS}

a)

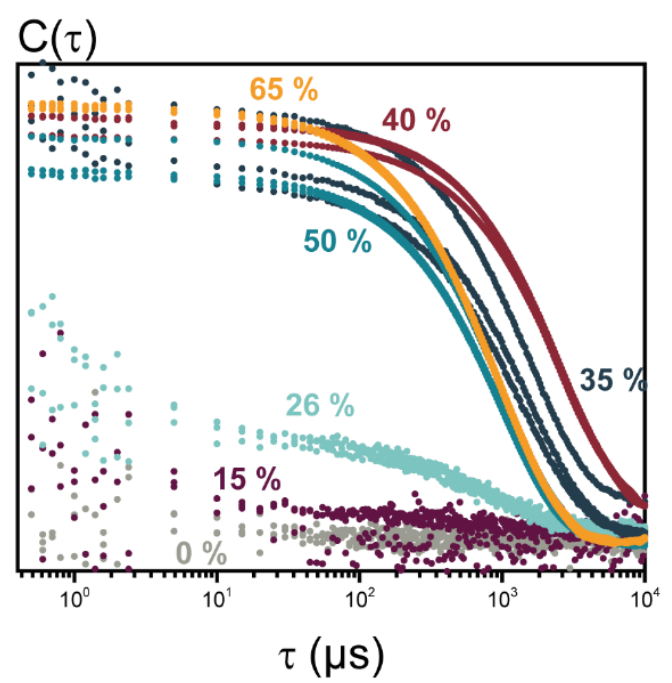

b)

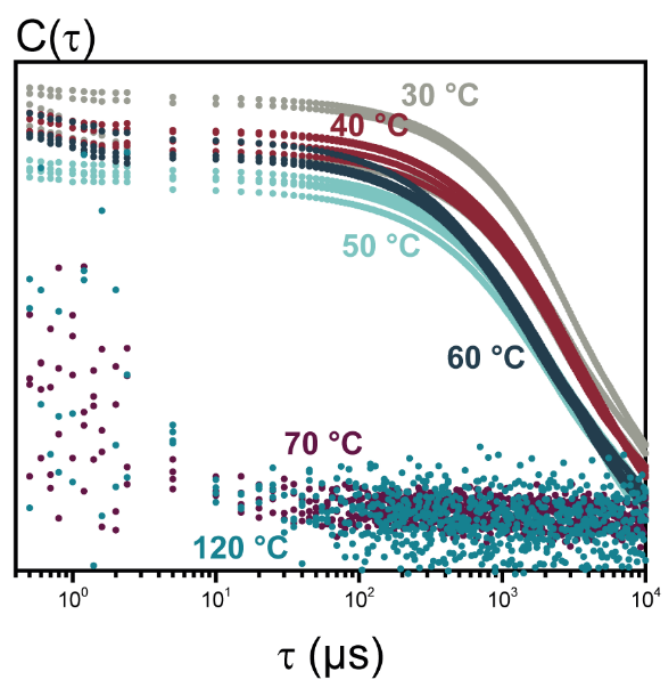

Figure S7: Original correlograms for DLS a) Solvent/non-solvent system b) temperature regime. 


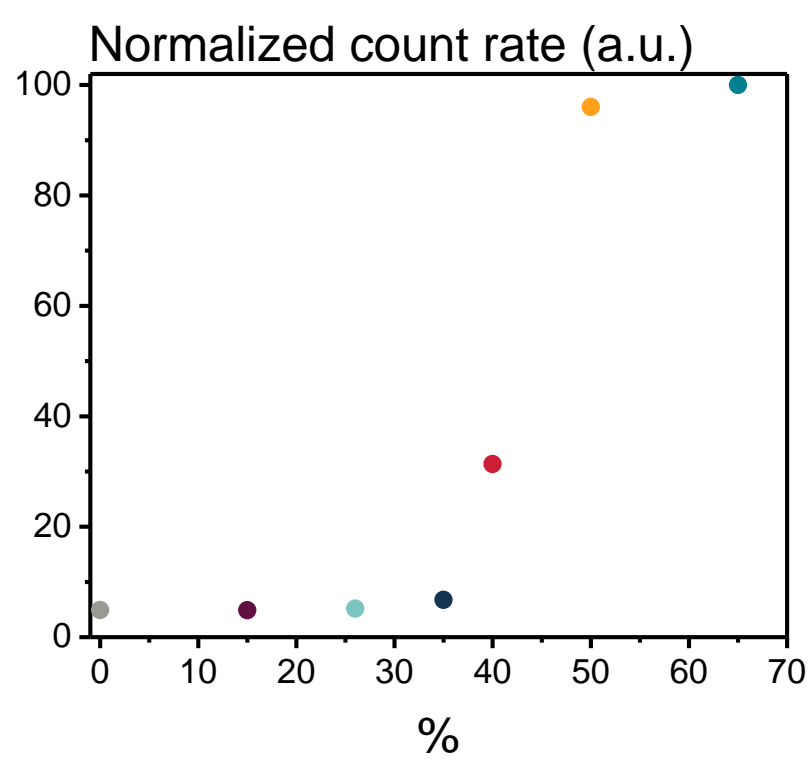

Figure S8: Normalized count rate for DLS scattered intensities for star-shaped P3AT in solvent/non-solvent system.

\section{AFM}

a)

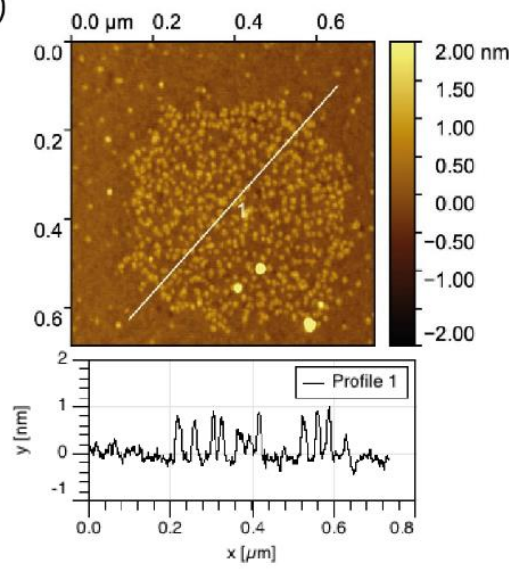

b)

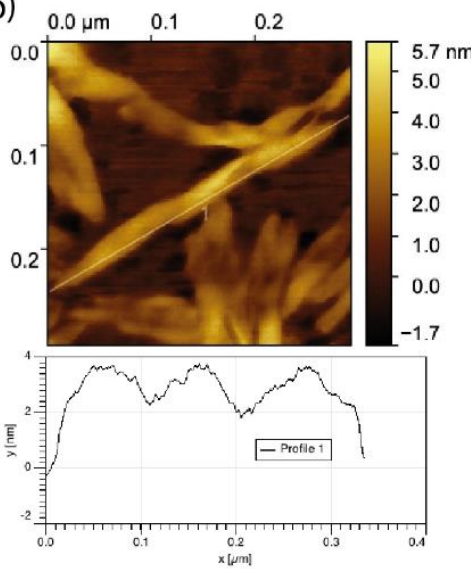

c)

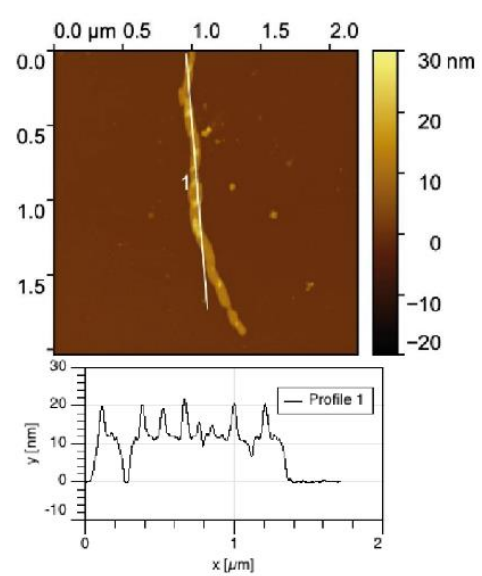

Figure S9: Detailed AFM images and cross section (along white line) of star-shaped P3AT a) in chloroform: globules of $1.5 \mathrm{~nm} \mathrm{~b}$ ) in 65\% methanol: well-formed twisted fibers of around $300-400 \mathrm{~nm} \mathrm{c}$ ) in hexanol at $40{ }^{\circ} \mathrm{C}$ : fibers (long and small) with a less regular structure.

\section{Supplementary References}

(1) Van Steerteghem, N.; Clays, K.; Verbiest, T.; Van Cleuvenbergen, S. Third-Harmonic Scattering for Fast and Sensitive Screening of the Second Hyperpolarizability in Solution. Anal. Chem. 2017, 89 (5), 29642971. https://doi.org/10.1021/acs.analchem.6b04429.

(2) Castiglioni, E.; Abbate, S.; Lebon, F.; Longhi, G. Ultraviolet, Circular Dichroism, Fluorescence, and Circularly Polarized Luminiscence Spectra of Regioregular Poly-[3-((S)-2-Methylbutyo)-Thiophene] in Solution. Chir 2012, 4324, 725-730. https://doi.org/10.1002/chir. 